\title{
Late Emergence of the Vibrissa Direction Selectivity Map in the Rat Barrel Cortex
}

\author{
Yves Kremer, ${ }^{1,2,3}$ Jean-François Léger, ${ }^{1,2,3}$ Dan Goodman, ${ }^{4,5}$ Romain Brette, ${ }^{4,5}$ and Laurent Bourdieu ${ }^{1,2,3}$ \\ ${ }^{1}$ Ecole Normale Supérieure, Institut de Biologie de l'ENS, IBENS, Paris, F-75005 France, ${ }^{2}$ Inserm, U1024, Paris, F-75005 France, ${ }^{3} \mathrm{CNRS}$, UMR 8197 , Paris, \\ F-75005 France ${ }^{4}$ Laboratoire Psychologie de la Perception, CNRS and Université Paris Descartes, Paris, F-75006 France, and ${ }^{5}$ Département d’Etudes \\ Cognitives, Ecole Normale Supérieure, Paris, F-75005 France
}

In the neocortex, neuronal selectivities for multiple sensorimotor modalities are often distributed in topographical maps thought to emerge during a restricted period in early postnatal development. Rodent barrel cortex contains a somatotopic map for vibrissa identity, but the existence of maps representing other tactile features has not been clearly demonstrated. We addressed the issue of the existence in the rat cortex of an intrabarrel map for vibrissa movement direction using in vivo two-photon imaging. We discovered that the emergence of a direction map in rat barrel cortex occurs long after all known critical periods in the somatosensory system. This map is remarkably specific, taking a pinwheel-like form centered near the barrel center and aligned to the barrel cortex somatotopy. We suggest that this map may arise from intracortical mechanisms and demonstrate by simulation that the combination of spike-timing-dependent plasticity at synapses between layer 4 and layer $2 / 3$ and realistic pad stimulation is sufficient to produce such a map. Its late emergence long after other classical maps suggests that experience-dependent map formation and refinement continue throughout adult life.

\section{Introduction}

Many cortical areas are organized into domains representing sensory parameters. Although their formation and their benefit for information processing are still poorly understood, it is often observed that primary sensory cortices are organized in several overlapping maps representing various parameters (Bartfeld and Grinvald, 1992), as e.g., in the cat and primate visual cortex. Evolution has endowed rodents with an efficient vibrissa tactile system, adapted to navigation in the absence of visual cues (Petersen, 2007). Neurons in SIbf (primary somatosensory cortex, barrel field) are known to have their activity modulated by numerous parameters. In addition to their tuning to the principal vibrissa identity (the barrel map), some neurons preferentially respond to specific directions of vibrissa deflections (Bruno et al., 2003; Lee and Simons, 2004), to high-frequency vibrations (Andermann et al., 2004), or to the apparent global motion of objects through the vibrissa pad (Jacob et al., 2008). However, no superposition of functional maps has been reproducibly observed in SIbf. Only one electrophysiological study has shown evidence for a second map in SIbf of adult rats, representing the vibrissa di-

Received Dec. 13, 2010; revised May 31, 2011; accepted June 6, 2011.

Author contributions: Y.K., J.-F.L., D.G., R.B., and L.B. designed research; Y.K., J.-F.L., D.G., R.B., and L.B. performed research; Y.K., J.-F.L., and L.B. analyzed data; Y.K., J.-F.L., R.B., and L.B. wrote the paper.

This work was supported by Human Frontier Science Program Grant RPG0060/2003-C102, by Agence Nationale pour la Recherche Grants NATACS ANR-07-NEUR-025-01 and PAMM 06-BLAN-0178-02, by the CNRS (ATIP 2003), by Région lle-de-France (E. 1762), and partially by the European Research Council (StG 240132). Y.K. was funded by the Ministère de l'Enseignement Supérieur of Luxembourg and the Fondation pour la Recherche Médicale. We are grateful to Boris Barbour, Daniel Shulz, Clément Léna, and Carl Petersen for the careful reading of the manuscript.

The authors declare no competing financial interests.

Correspondence should be addressed to Laurent Bourdieu, Institut de Biologie de I'ENS, IBENS, 46 rue d'Ulm, 75005 Paris, France. E-mail: laurent.bourdieu@ens.fr.

DOI:10.1523/JNEUROSCI.6541-10.2011

Copyright $\odot 2011$ the authors $\quad 0270-6474 / 11 / 3110689-12 \$ 15.00 / 0$ rection selectivity (Andermann and Moore, 2006). However a subsequent two-photon imaging study has recently established that this second map was absent in 1-month-old rats (Kerr et al., 2007). One possibility for interpreting these opposite results could be the role played by the age of the animals, but assessing it is precluded by the difficulty of comparing electrophysiological results with low neuronal spatial sampling and the exhaustive mapping of biphotonic microscopy.

As sensory experience is known to play an important role in the maturation of sensory functional maps, as e.g., orientation or direction selectivity in the visual cortex (Crair et al., 1998; Li et al., 2008), our aim is to understand whether increased sensory experience could facilitate the emergence of the barrel direction selectivity map and to explore how experience could link the architecture of this map with the underlying barrel map. Two-photon calcium imaging was used to record, in rats of different ages, large-scale neuronal activity with single-cell resolution and the position of all recorded cells. We show that juvenile animals (4-6 weeks old) do not possess a coherent direction tuning map, but such a map is observed in adult animals (12-16 weeks old). A related map emerges in the neuropil, with sharp frontiers separating domains with opposite direction selectivities.

This late maturation of the directional map takes place after most other stages of development of the rat nervous system (Feldman and Brecht, 2005; Inan and Crair, 2007; Petersen, 2007). The fact that it appears late and demonstrates somatotopic order emphasizes the possible role of sensory experience in its formation. We propose a model linking the repeated presentation of objects crossing the vibrissa pad and the emergence of a direction selectivity map, based on the architecture of SIbf and a spike-timingdependent plasticity (STDP) learning rule. Beyond the formation of maps with patterns governed by genetically encoded processes, 
a

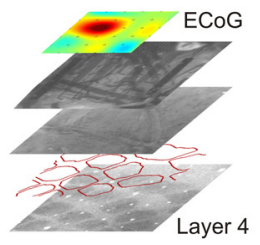

b

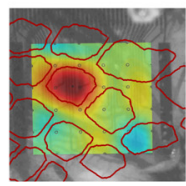

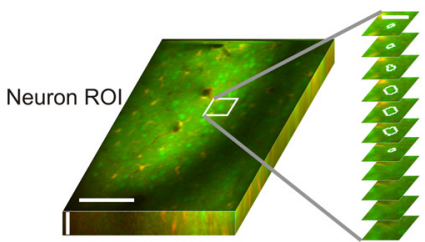

C

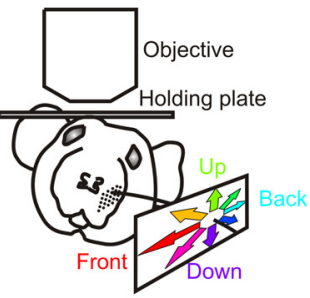

d

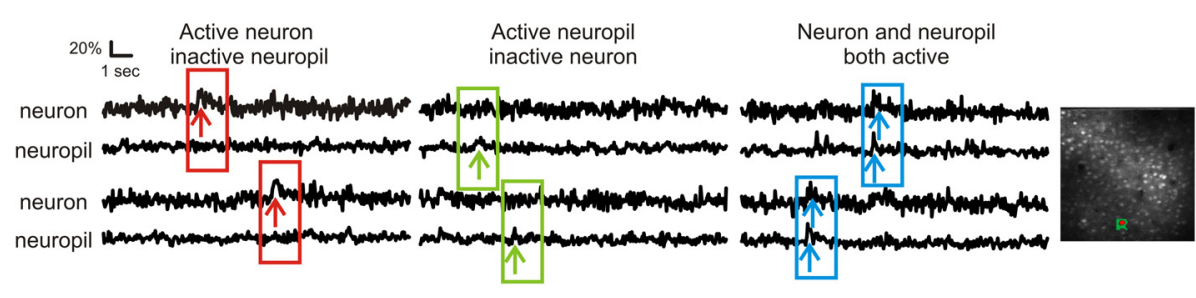

e

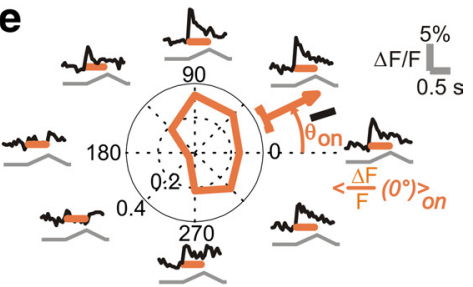

f

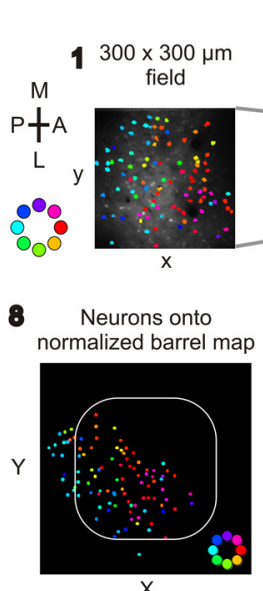

X

\section{$2680 \times 680 \mu \mathrm{m}$}

$8 \begin{aligned} & \text { Project vertical blood } \\ & \text { vessels onto layer } 4\end{aligned}$
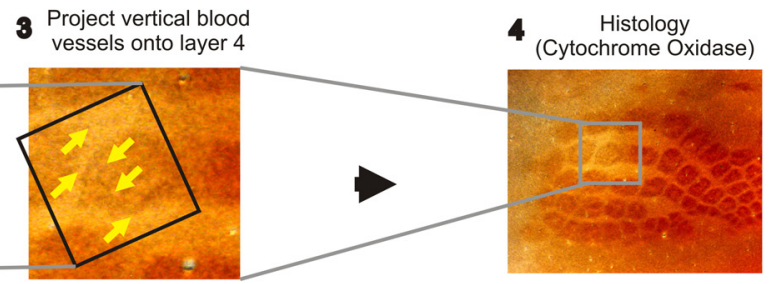

6 Normalized barrels coordinates

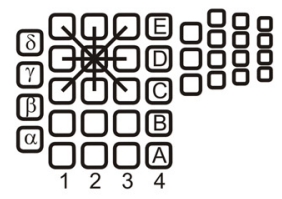

6 Barrels

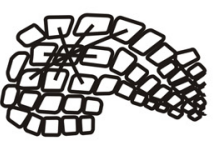

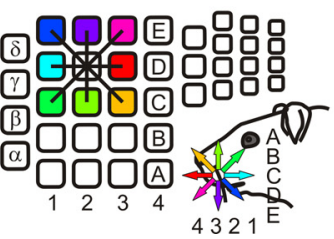
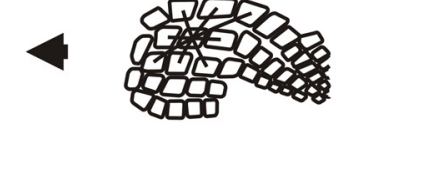

Figure 1. a, Barrel column localization with a microelectrode array. Left, Montage showing an electrocorticogram (ECOG) $10-15$ ms after a vibrissa deflection, a bright-field image of the brain surface, a layer $2 / 3$ section of the underlying cortex, and a cytochrome oxidase-stained section of layer 4 with a drawing of the barrel contours. Right, Superposition of these images. $\boldsymbol{b}$, Two-photon image stack in an adult rat. Right, Zoom showing the horizontal sections of a neuron in the center of the stack (white outlines). Scale bars: vertical, $30 \mu \mathrm{m}$; horizontal, $100 \mu \mathrm{m} ; z 00 \mathrm{med}, 15 \mu \mathrm{m}$. c, Color code for the deflection direction of an individual vibrissa. $\boldsymbol{d}$, Examples of simultaneous or independent calcium transients in individual recordings of a neuron and its surrounding neuropil. Epochs with calcium transient are highlighted in a rectangle and the transient maximum is marked with an arrow. In each case, two examples are shown. Left, The neuron only is active. Middle, The neuropil only is active. Right, The neuron and the neuropil are both active. Inset, Image showing the corresponding neuron (red, preferred direction $2.2^{\circ}$ ) and its surrounding neuropil patch (green, preferred direction $142.9^{\circ}$ ). This field is shown in the Figure $2 a$, right. $\boldsymbol{e}$, Directional responses of a typical adult neuron. Black traces, Average fluorescence responses ( $N=150$ stimulations) of the neuron (shown in $\boldsymbol{b}$ ) to deflections in the eight cardinal directions. Gray traces, Deflection time courses. Orange segments, Time windows used for integrating the neuronal responses $\langle\Delta F / F\rangle_{O N}$ shown as a tuning curve in the center. Orange arrow, Preferred direction $\theta_{0 N}$ with a length proportional to the TR (black scale bar, 0.25$)$. Orange circular arc, Uncertainty $\sigma_{\theta}$ for $\theta_{0 \mathrm{~N}} . \boldsymbol{f}$, Pooling of direction selectivity maps from different fields and barrels. 1 , Neuron Cartesian coordinates $(x, y)$ in the image. 2, Localization of vertically oriented radial blood vessels (yellow arrows) from two-photon images $(680 \times 680 \mu \mathrm{m}) .3,4$, Identification of barrel identity and contours from a flattened layer 4 slice stained with cytochrome oxidase. $\mathbf{5 , 6}$, Normalized barrel coordinates calculated by $y$-axis shearing $\left(0-30^{\circ}\right.$ depending on barrel identity) such that the horizontal axis joining adjacent barrels in a row became orthogonal to the vertical axis joining adjacent barrels in an arc. 7, Adjacent barrels are represented with the color attributed to principal barrel neurons whose preferred direction corresponds to a deflection toward these adjacent vibrissae, to keep the color legend aligned with adjacent barrels (head, inset). 8 , Neuron normalized coordinates $X, Y$, with values of +1 and -1 indicating barrel borders intersected by row and arc axes in anteroposterior and mediolateral directions, respectively.

our model underlines the link that may exist between plasticity mechanisms and the formation of functional maps.

\section{Materials and Methods}

Surgery. All surgical procedures were in accordance with the European Community guidelines on the care and use of animals (86/609/CEE, CE official journal L358, 18th December, 1986), French legislation (decree no. 97/748, 19th October, 1987, J. O. République française, 20th October, 1987), and the recommendations of the CNRS. Four juvenile (P32-P39; weight $\sim 100 \mathrm{~g}$ ) and seven adult (P81-112; weight $\sim 350 \mathrm{~g}$ ) male Sprague Dawley rats were included in this study. Before the experiment, all rats were held in standard cages and in groups to enhance the use of their vibrissae in a social environment. On the day of the experiment, rats were anesthetized with isoflurane (1-1.5\%) in $\mathrm{O}_{2} / \mathrm{N}_{2} \mathrm{O}(20 \% / 80 \%)$, placed onto a heating blanket and maintained in a stereotaxic frame using a custom-made mouth piece (courtesy of Daniel Shulz, UNIC, Gif-sur-
Yvette, France) and mask allowing complete access to the vibrissae. Heartbeat and breathing were continuously monitored. A craniotomy $(\sim 3 \mathrm{~mm}$ in diameter) centered at $-2.5 \mathrm{~mm}$ from bregma and $+5.5 \mathrm{~mm}$ laterally was performed on the rat's right hemisphere and the dura mater was removed. Throughout the experiment, the brain was bathed in external saline (in mм: $125 \mathrm{NaCl}, 2.5 \mathrm{KCl}, 26 \mathrm{NaHCO}_{3}, 1.25 \mathrm{NaH}_{2} \mathrm{PO}_{4}, 2$ $\mathrm{CaCl}_{2}, 1 \mathrm{MgCl}_{2}$, and 20 glucose).

Microelectrocorticogram recordings. To target the dye injection, individual barrels were identified by electrophysiological surface recordings using flexible 32-electrode arrays (FlexMEA-300/30, MultiChannel Systems) after dura mater removal. By averaging 10-20 responses to individual vibrissa deflections, and using a spline interpolation between neighboring electrodes, the barrel center was localized with a precision of at least $\pm 50 \mu \mathrm{m}$ (Fig. 1a).

Two-photon microscopy. Multicell bolus loading of Oregon-Green BAPTA 1-AM (OGB1-AM) was performed according to the protocol described by 
Stosiek et al. (2003) (Fig. 1b). A $10 \mu$ d drop of sulforhodamine 101 (SR101; $100 \mu \mathrm{M}$ in external saline) was deposited onto the brain surface to stain specifically astrocytes (Nimmerjahn et al., 2004). The craniotomy was filled with agarose gel $(\sim 1.5 \%$; type III-A, Sigma-Aldrich) and sealed with a glass coverslip. Fluorescence was monitored with a custom in vivo microscope coupled with a Mai-Tai (Spectra Physics) mode-locked Ti:sapphire laser $(840 \mathrm{~nm}$ or $900 \mathrm{~nm})$. Fields of view of 150,200 , or $300 \mu \mathrm{m}$ were scanned at 10 or $20 \mathrm{~Hz}$ using a combination of a resonant scanner (CRS-Series, GSI) and a galvanometric scanner (M-Series, $9 \times 20 \mathrm{~mm}$, GSI). To improve the imaging depth while preserving a high optical axial sectioning, an effective numerical aperture (NA) smaller than the objective full NA was used (Dunn et al., 2000; Helmchen and Denk, 2005). The beam diameter at the objective (Olympus XLum 20×, NA 0.95) back focal plane was adjusted to obtain a fill factor of its back aperture of $\sim 0.7$ and a resulting effective NA of $\sim 0.65$ (Helmchen and Denk, 2005). It provided an axial resolution of $3.5 \pm 0.5 \mu \mathrm{m}$ and an in-plane resolution of $0.8 \pm 0.05 \mu \mathrm{m}$ for excitation at $900 \mathrm{~nm}$. Fluorescence detection was achieved with a digital photomultiplier (PMT) module (H7421-40, Hamamatsu) (Wier et al., 2000; Benninger et al., 2008). Coordinates from the sinusoidal resonant scan were linearized before further analysis. Before a recording session for a given field, a green (OGB1$\mathrm{AM}$ ) and a red (SR101) $z$-stack (referred as reference stacks) were acquired around the plane of interest ( $\pm 15 \mu \mathrm{m} ; 3 \mu \mathrm{m}$ between planes) (Fig. $1 b$ ). Recordings were acquired in the upper part of the layers $2 / 3$, from $150 \mu \mathrm{m}$ below the brain surface down to $300 \mu \mathrm{m}$.

Vibrissa stimulation. A pair of crossed linear ultrasonic closed-loop piezomotors (M-663 PI-Line linear plates; Physik Instrumente, PI) provided vibrissa stimulation in two dimensions (Fig. 1c). The vibrissa was inserted into a glass capillary fixed onto the translation device and glued with wax at a distance of $5 \mathrm{~mm}$ from the mystacial pad. Great care was taken to align the stimulator with the axis of the vibrissa and not to displace the vibrissa from its resting position. Control measurements were performed to verify that our vibrissa stimulation did not produce a displacement of the adjacent vibrissae by pure facial skin conduction (Micro- $\varepsilon$ OptoNCDT 1700). In juvenile and adult animals, the measured adjacent vibrissa displacements were below the threshold for eliciting spikes in the trigeminal ganglion [always below velocities of $1 \%$ s (Gibson and Welker, 1983)]. We also checked that there was no bias for any direction of stimulation in terms of amplitude and rise time (also measured with OptoNCDT 1700). All stimuli consisted of a saw-tooth pattern of $500 \mu \mathrm{m}$ in amplitude and lasting $1 \mathrm{~s}$. Two different time courses were used. The first stimulus consisted of an ON ramp lasting for $500 \mathrm{~ms}$ followed by an OFF ramp lasting $500 \mathrm{~ms}$. The second consisted of an ON ramp lasting $20 \mathrm{~ms}$ and an OFF ramp lasting 980 ms. Stimuli were separated by a $1 \mathrm{~s}$ blank and grouped in blocks of eight, each stimulus deflecting the vibrissa along one of the eight cardinal directions (in an order kept constant throughout an entire protocol but varied from protocol to protocol). Individual blocks were separated by $4 \mathrm{~s}$, the time required for data storage [duration of one block $=8 \times(1 \mathrm{~s}$ stimulus $+1 \mathrm{~s}$ blank $)+4 \mathrm{~s}$ of data storage $=20$ s]. For each field of view, a total of 1200-1600 stimuli (150-200 blocks) were presented. It therefore took between 50 and $66 \mathrm{~min}$ to acquire one field of view.

Direction selectivity calculation. Individual cell bodies were automatically detected in 3D from the OGB1-AM and SR101 reference stacks using a custom-written algorithm (Matlab) that used the SR101 staining to discriminate neuronal somata and astrocytes. For each block of eight successive stimulations, an averaged image of the field was computed and compared to the stack to determine its depth. For a given neuron, the eight stimulations of a block were included in the analysis only if the depth of the corresponding average image was within $\pm 3 \mu \mathrm{m}$ of the neuron equatorial horizontal plane. This criterion minimizes the risk of contamination between the neuropil and the neuronal signal in the $z$ direction. In the $x-y$ plane, contamination was also minimized by defining regions of interest (ROIs) for neurons slightly smaller than the image of their soma and, for neuropil direction selectivity, by excluding neuronal ROI slightly larger than the soma from the surrounding neuropil surface. Neuronal and surrounding neuropil signals were well separated because calcium transients often occurred in only one of the two signals (Fig. 1d) (Göbel and Helmchen, 2007). Fluorescence signals were first corrected for the nonlinearity of the photon counting at high incident photon rate, due to the finite Pair-Pulse Resolution
(53 ns) of the PMT (Wier et al., 2000) and were then summed for each ROI. For each neuron, fluorescence signals were averaged $\left(\overline{F_{k}}\right)$ over all stimulations sharing a same direction $\varphi_{k}$ (example of a typical neuron in Fig. 1e: each direction averaged over 150 repetitions). This average had two effects: it reduced the photon noise and it smoothed the stochastic variability of the neuron to any stimulation. Then, the relative fluorescence variation $\frac{\overline{\Delta F_{k}}}{\overline{F_{k, 0}}}=\frac{\overline{F_{k}}-\overline{F_{k, 0}}}{\overline{F_{k, 0}}}$ was computed with respect to the baseline fluorescence $\overline{F_{k, 0}}$ defined as the mean fluorescence during the $500 \mathrm{~ms}$ preceding the onset of the stimulus. The neuron response amplitude $\left\langle\frac{\Delta F}{F}(k)\right\rangle$ to that direction was finally computed as the mean of $\frac{\overline{\Delta F_{k}}}{\overline{F_{k, o}}}$ during the first 500 ms following onset of a 500-ms-long ON ramp, or the last $500 \mathrm{~ms}$ of a 980-ms-long OFF ramp (in this second case, only fluorescent variations after an $\sim 500 \mathrm{~ms}$ delay following the change in direction were taken into account to prevent any contamination between $\mathrm{ON}$ and OFF ramps). In the case of the ON ramp, after measuring the neuron responses to the eight cardinal directions $\varphi_{k}=k(2 \pi / 8)(k=0-7)$, its preferred direction $\theta_{\mathrm{ON}}$ was calculated as the angle of the vector $R_{\mathrm{ON}}=\Sigma_{\varphi_{k}=0^{\circ} . .315^{\circ}}\left\langle\frac{\Delta F}{F}\left(\varphi_{k}\right)\right\rangle_{\mathrm{ON}} \cdot e^{j \varphi_{k}}$, where $j^{2}=$ -1 . The tuning selectivity was assessed by the tuning ratio,

$$
\mathrm{TR}_{\mathrm{ON}}=\frac{\left|R_{\mathrm{ON}}\right|}{\sum_{\varphi_{k}=0^{\circ} \ldots 315^{\circ}}\left\langle\frac{\Delta F}{F}\left(\varphi_{k}\right)\right\rangle_{\mathrm{ON}}}
$$

Estimation of a neuron preferred direction was alternatively also achieved using the 980 -ms-long OFF ramps. Corresponding $\theta_{\mathrm{OFF}}, R_{\mathrm{OFF}}$, and $\mathrm{TR}_{\mathrm{OFF}}$ were calculated. We verified that preferred directions were unchanged when they were estimated either from ON or OFF ramps. Results obtained in ON and OFF phases are pooled in the rest of this article.

Quantification of the statistical significance of neuron response anisotropy was achieved by a bootstrap method. Responses to each direction were reaveraged over a random sample of 100 stimulations drawn with replacement from the original stimulations, and a bootstrap resultant complex sum $R^{\star}$ was computed. This process was repeated 200 times to evaluate the standard deviation $\sigma_{R}$ of the distribution of the $\left|R^{\star}\right|$. The directional selectivity of the neuron was considered as being sufficiently characterized when $|R|$ exceeded $1.6452 \sigma_{R}$, meaning that the probability to be isotropic had fallen below 0.1 [threshold used for comparison with previous two-photon study (Kerr et al., 2007)]. The standard deviation $\sigma_{\theta}$ of the distribution of angle of the bootstrap $R^{\star}$ was also calculated to estimate the uncertainty in the preferred direction of a neuron.

Histology, two-photon image registration, and pooling of maps from different fields and barrels. Figure 1 fillustrates the registration procedure. At the end of the experiments, rats were deeply anesthetized and perfused in $4 \%$ paraformaldehyde. Slices tangential to the cortical surface were cut and stained with cytochrome oxidase, and barrel contours were identified visually. Barrel centers were defined as the barycenters of the surfaces delimited by these contours. Radial blood vessels (perpendicular to the slices) were used for registration of the two-photon images and electrocorticogram with respect to the barrel center and borders with $\pm 50 \mu \mathrm{m}$ accuracy. Normalized barrel coordinates were calculated by shearing the $y$-axis $\left(\sim 0-30^{\circ}\right.$, depending on barrel identity) such that the horizontal axis joining the adjacent barrels' centers in the same row became orthogonal to the vertical axis joining the adjacent barrels' centers in the same arc. As recordings were performed in SIbf contralateral to the stimulation side, an axial symmetry was applied compared to the direction on the periphery to keep the color legend aligned with adjacent barrels. Neuron coordinates were finally normalized such that $X$ and $Y$ values of +1 and -1 indicated barrel borders intersected by row and arc axes in anteroposterior and mediolateral directions, respectively.

Map analysis. The mean correlation $\langle g\rangle=\left\langle T R_{i} \cdot T R_{j} \cdot\right.$ $\left.\cos \left(\theta_{i}-\theta_{j}\right)\right\rangle_{(i, j)}$ was calculated for three different types of neighbor 
pairs $(i, j)$. A Voronoi segmentation of the significantly anisotropic neurons in a field or in a superposition of fields was used to define unambiguously first-neighbor pairs or second-neighbor pairs. The third type of neighbor pairs was the family of pairs that could be formed between one neuron and all significantly anisotropic neurons within a given radius $\rho$ ( $\rho=$ $50,100,150$, or $200 \mu \mathrm{m})$.

To analyze the spatial organization of maps, each neuron was described by its polar angular coordinate $\alpha$ and its preferred direction $\theta$. Pinwheel center optimization was achieved by choosing the origin of neuronal position in polar coordinate $\alpha$ at different locations in the barrel section. For each origin position, we tested the model $\theta=\alpha+\alpha_{0}\left(\operatorname{modulo} 360^{\circ}\right)$ and calculated the probability of not observing such linear association using the statistic described by Fisher and Lee $(1983,1986)$.

The Gaussian filtering procedure used to smooth maps of preferred direction was the following: at each position $\left(x_{0}, y_{0}\right)$ of a barrel section, we defined a complex value called the local preferred mean director by

$$
\bar{\Lambda}=\frac{\sum_{n} \mathrm{TR}_{n} \cdot e^{-\frac{\left(x_{0}-x_{n}\right)^{2}+\left(y_{0}-y_{n}\right)^{2}}{2 \omega^{2}}} \cdot e^{j \theta_{n}}}{\sum_{n} \mathrm{TR}_{n} \cdot e^{-\frac{\left(x_{0}-x_{n}\right)^{2}+\left(y_{0}-y_{n}\right)^{2}}{2 \omega^{2}}}},
$$

a

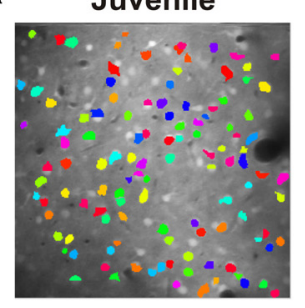

c

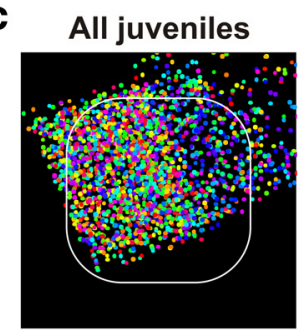

e

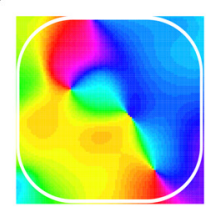

All juveniles

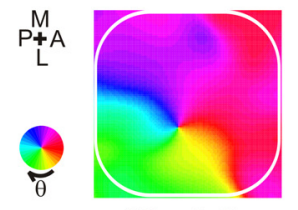

All adults
Adult
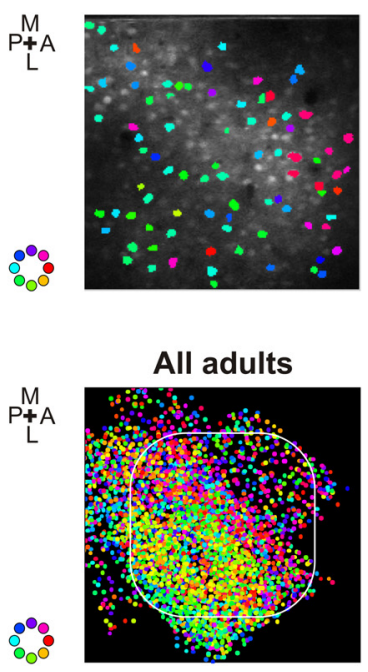

$\mathbf{f}$
Figure 2. Age dependence of the direction selectivity map. $\boldsymbol{a}$, Example of direction selectivity organization in a juvenile rat (left) and in an adult rat (right). Each dot corresponds to one neuron. The color code is inverted compared to the arrow order in Figure 1c: it is oriented such that a movement toward a neighboring vibrissa is represented as a vector pointing toward this vibrissa barrel $(M$, medial; $L$, lateral; $P$, posterior; $A$, anterior). $\boldsymbol{b}$, Mean correlations between neighboring neurons in the fields shown in $\boldsymbol{a}$. Asterisks indicate statistically significant differences between the juvenile and adult rats $(p<0.001)$. $c$, Superposition of all recorded neurons in normalized barrel coordinates for juvenile rats (left, $N=4$ animals, 29 fields, barrels: $\mathrm{C2}, \mathrm{C} 3, \mathrm{D} 2, \mathrm{D} 3)$ and for adult rats [right, $N=7$ animals, 41 fields, barrels: B1, B2, C2, D2 (×3), D3]. White contour lines indicate normalized barrel borders. $\boldsymbol{d}$, Mean correlations between neighboring neurons in all fields recorded in juveniles (black, $N=29$ fields) and in adults (white, $N=41$ fields). All comparisons between ages are significantly different (asterisks, $\mathrm{P}<0.001$ ). $e$, Direction selectivity maps smoothed with a Gaussian filter (half-width $\omega=0.2$ in normalized barrel coordinates). $\boldsymbol{f}$, Direction selectivity smoothed maps scaled with color saturation proportional to the local homogeneity index. called the local homogeneity index (LHI) which measured how anisotropic and collinear the neurons (or neuropil patches) were locally. Low LHI corresponds to regions with weak correlation between neuron (or neuropil) preferred directions.

After automatic detection of neuronal somata and astrocytes, the neuropil was defined as the remaining volume, excluding blood vessels. Neuropil was then segmented into patches of $25 \mu \mathrm{m} \times 25 \mu \mathrm{m}$. For each of these ROIs, a preferred direction and a tuning ratio were computed as for neurons. Neuron-neuropil correlation corresponds to the correlation of preferred directions between a neuron and its closest neuropil patch.

To quantify the existence of sharp frontiers between neuropil domains, the metric $\Delta$ was introduced. A straight frontier can be viewed as the solution to the geometrical problem of the minimization of the interface cost between two juxtaposed domains. Therefore, we can evaluate the presence of a neuropil frontier in a field by calculating a metric $\Delta$ defined as the gain of correlation between first-neighbor neuropil patches in the raw data, compared to the correlation measured if all neuropil patches were spatially shuffled. More precisely, $\Delta$ was constructed as follows: for a given field, each significantly $(p<0.1)$ anisotropic neuropil patch $i$ was correlated with those of its eight closest neighbors, which were also significantly anisotropic:

$$
g_{i}=\frac{\sum_{\text {neighbors }(j)} \cos \left(\theta_{i}-\theta_{j}\right)}{\sum_{\operatorname{neighbors}(j)} 1},
$$

and all $g_{i}$ were averaged for this field $\left(\bar{g}^{\text {data }}\right)$. A first shuffled control was obtained by random permutation between significantly anisotropic neuropil

patches of their preferred directions and a $\bar{g}_{1}^{\text {shuffled }}$ was calculated by analogy with $\bar{g}$ data but with the permuted directions. A collection of 200 similar shuffled controls was computed and averaged to form $\bar{g}_{\text {mean }}^{\text {shuffled }}=$ $(1 / 200) \sum_{k} \bar{g}_{k}^{\text {shuffled }}$. Finally we obtained $\Delta$ by subtracting: $\Delta=\bar{g}^{\text {data }}-\bar{g}_{\text {mean }}^{\text {shufled }}$.

Network simulations. The numerical model of the barrel cortex network was written with the Brian simulator (Goodman and Brette, 2009). It included $5 \times 5$ vibrissae, 1 barrel per vibrissa, arranged as a square grid, 31,325 neurons and $\sim 6,000,000$ synapses. Stimulations consisted of bars (one barrel wide) with random orientation swept across the vibrissa pad at a speed of 1 barrel per $5 \mathrm{~ms}$. After a sweep, no stimulus was presented for $55 \mathrm{~ms}$. Simulations lasted $1 \mathrm{~h}$ (biological time), corresponding to 65,000 stimuli. In Figure $7 e$, bar directions were drawn at random with probability density $p(\theta)=[1+(1 / 2) \cos (2 \theta)]^{k}$, where $k$ is a parameter controlling the degree of rostrocaudal bias: $k=0$ corresponds to a uniform distribution, $k=\infty$ corresponds to only rostrocaudal directions $\left(\theta=0^{\circ}\right.$ and $\left.180^{\circ}\right)$.

Layer 4 contained $22 \times 22$ neurons per barrel (12,100 neurons). L4 neurons fired according to a Poisson process with a firing rate $F(\theta)=$ $\left(F_{\max } / 2\right)\left[\cos \left(\theta-\theta^{*}\right)+1\right]$, where $\theta^{*}$ is the preferred direction of deflection (no firing in the absence of stimulus). Preferred direction was random and $F_{\max }$ was chosen so that a neuron fired on average 0.5 spike in response to a $5 \mathrm{~ms}$ deflection at its optimal direction (i.e., $F_{\max }=100$ $\mathrm{Hz}$ ). Layer $2 / 3$ was composed of $25 \times 25$ excitatory and $12 \times 12$ inhibitory neurons per barrel (15,625 excitatory and 3600 inhibitory neurons). L2/3 neurons were integrate-and-fire models with adaptation and refractory period of $2 \mathrm{~ms}$. Adaptation was modeled by an increase in spike threshold of $2 \mathrm{mV}$ after each spike, followed by an exponential decay to $V_{\mathrm{T}}=-55 \mathrm{mV}$ with a time constant of $50 \mathrm{~ms}$. The resting potential 

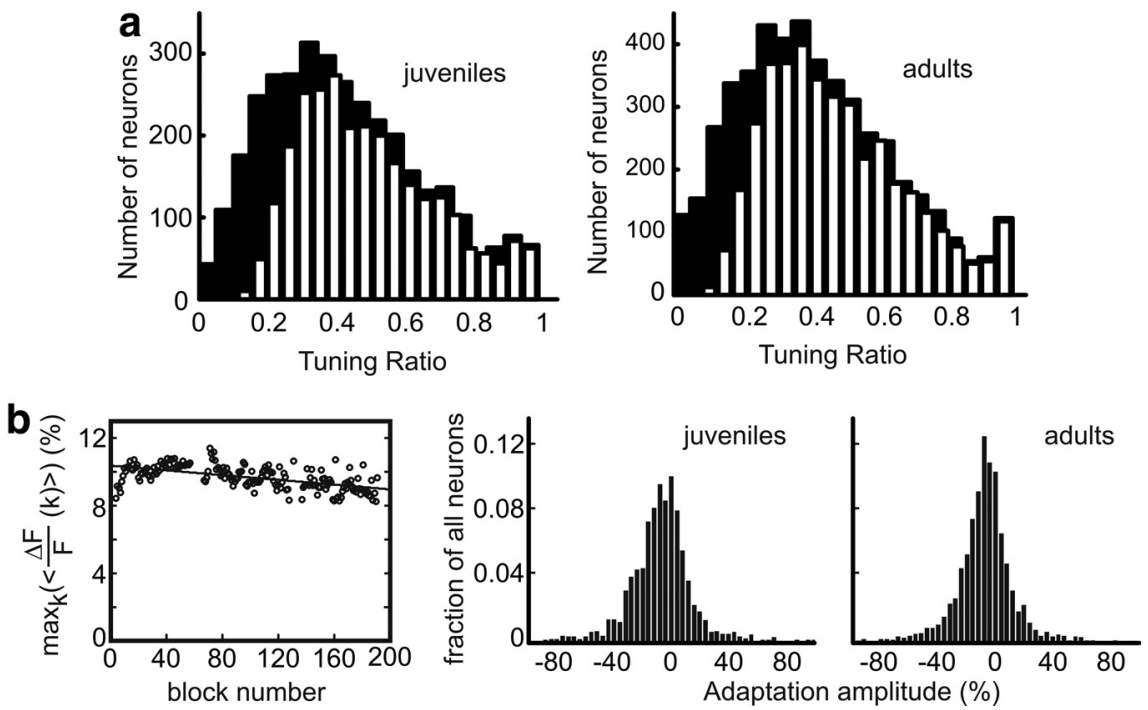

Figure 3. Juvenile and adult tuning and adaptation properties of neurons. $\boldsymbol{a}$, Histogram of tuning ratio of all the neurons recorded in the juvenile animals (left) and in adult ones (right). Black bars, All neurons; white bars, statistically significantly tuned neurons. $\boldsymbol{b}$, Adaptation of the neuronal response across blocks. Left, Typical evolution of the response amplitude of a juvenile neuron. The maximum response for each block of eight different directions $\max _{k}(\langle(\Delta F / F)(k)\rangle)$ is plotted as a function of the block number. A slight continuous adaptation is observed. Right, Normalized histogram of the adaptation amplitude, defined as the relative variation (in percentage) of $\max _{k}(\langle(\Delta F / F)(k)\rangle)$ during the protocol (the first 150 blocks of stimulation) for juvenile and adult animals.

was $E_{\mathrm{L}}=-70 \mathrm{mV}$. L2/3 neurons received a sum of EPSPs and IPSPs, modeled as $\beta$ functions (difference of two exponential functions). EPSPs had time constants of 2 and 10 ms (Feldmeyer et al., 2002; Brecht, 2007; Sarid et al., 2007), and IPSPs 10 and $25 \mathrm{~ms}$, giving peak times of 4 and 15 ms, respectively (Sun et al., 2006; Kapfer et al., 2007; Helmstaedter et al., 2009).

Synapses from L4 to L2/3 excitatory neurons (3,800,000 synapses) were modeled as follows. Neurons in L4 projected onto excitatory neurons in the same barrel in L2/3 with $50 \%$ probability (each pair had $50 \%$ probability of being connected). There were no synapses between neurons of different barrels in layer 4 . Those synapses were plastic (see below) with maximum weight (EPSP max) of $1 \mathrm{mV}$ (Feldmeyer et al., 2002; Brecht, 2007; Lübke and Feldmeyer, 2007; Sarid et al., 2007; Lefort et al., 2009). For each neuron in L2/3, we chose an initial preferred direction randomly and set the synaptic weight of all presynaptic neurons with preferred direction within $\pm 90^{\circ}$ at $1 \mathrm{mV}$ (the weight of all other synapses was $0 \mathrm{mV}$ ). There were 1,300,000 synapses between L2/3 excitatory and L2/3 excitatory neurons. The probability that two excitatory neurons in L2/3 were connected was a Gaussian function of their distance, with a standard deviation of 0.4 (in units of barrel width) and a maximum probability of $15 \%$ (at zero distance) (Petersen and Sakmann, 2001; Lübke and Feldmeyer, 2007). EPSPs were $0.3 \mathrm{mV}$ in amplitude (Feldmeyer et al., 2006; Brecht, 2007; Lübke and Feldmeyer, 2007; Lefort et al., 2009). L2/3 excitatory neurons connected also to inhibitory neurons with the same connectivity pattern, but EPSPs were $1 \mathrm{mV}$, causing a higher firing rate for inhibitory neurons (300,000 synapses in total between L2/3 excitatory to L2/3 inhibitory neurons). Finally, the probability that an inhibitory neuron projected onto an excitatory neuron in L2/3 was a Gaussian function of their distance, with a standard deviation of 0.2 (in units of barrel width) and maximum probability of $100 \%$ (at zero distance) (Helmstaedter et al., 2009) and IPSPs were $1 \mathrm{mV}$ high (530,000 synapses between L2/3 inhibitory and L2/3 excitatory neurons) (Sun et al., 2006).

STDP was modeled as by Song et al. (2000): the LTP time constant was $5 \mathrm{~ms}$ and the maximum potentiation was $0.5 \%$ of the maximum synaptic weight (Jacob et al., 2007). The LTD time constant was $25 \mathrm{~ms}$ and the maximum depression was $0.4 \%$ of the maximum synaptic weight (Jacob et al., 2007).

\section{Results \\ Spatial homogeneity of preferred direction increases with age}

To map extensive sections of barrel columns, fields of $300 \times 300 \mu \mathrm{m}$ were scanned. Figure $2 a$ illustrates two typical fields intersecting the central axis of the barrel columns, obtained from animals of different ages. The spatial organization of preferred directions showed a striking difference: for the juvenile rat (5 weeks old), neighboring neurons exhibited preferred directions with poor coherence, resulting in disordered patterns (Fig. 2a, left), whereas the adult rat (16 weeks old) showed much smoother transitions between preferred directions of neighboring neurons, forming more homogeneous domains (Fig. 2a, right). This difference was quantified by the local correlation of direction preferences $\langle g\rangle$, which measures how similar angular preferences are found among neighbors (Fig. 2b), taking into account either the first, second, or all neighbors within distances from 50 to $200 \mu \mathrm{m}$ (Materials and Methods). We observed in all cases a highly significant difference of local order between the two fields shown in Figure $2 a$ ( $t$ test, $p<0.001$ in all cases). Adult local order was significantly stronger than in juveniles and was the only one to depart significantly from zero correlation $(p<0.001$ in adult but $p>0.1$ in juvenile). It reveals the emergence of a local order in adult animals that is absent in juvenile rats.

\section{Microcolumnar organization of direction selectivity maps in adult rats}

By referencing every optically recorded field with postmortem histological images, normalized barrel coordinates were attributed to each neuron, which allows evaluation of the average organization on several barrels (Fig. 1f, Materials and Methods). All recorded fields in juvenile ( $N=4$ rats $)$ and adult $(N=7$ rats $)$ animals were thus pooled together (Fig. 2c). All fields were acquired in the upper part of layers $2 / 3$ at depths ranging from 150 to $300 \mu \mathrm{m}$ below the brain surface. Whereas spatial correlation of direction preferences was always apparent in adults, the average juvenile map did not exhibit a clear spatial segregation of the different direction preferences. For all fields included in these maps, local correlations were always significantly different between juvenile and adult animals $(p<0.001)$ (Fig. $2 d)$. To determine the spatial extent of the domains within which neurons have correlated preferred directions, we applied a spatial Gaussian low-pass filter to our data (Fig. 2e). In the adult map, structures with a spatial scale larger than the Gaussian kernel emerged. In Figure $2 f$, the same filtered maps were plotted with color saturation proportional to the local homogeneity index, a metric measuring how neurons share locally the same preferred direction (LHI, Materials and Methods). As expected, such averaging reduced the local disorder in all maps and showed that only adult rats possess a coherent average map organized in microdomains with high LHI revealing a dominant preferred direction.

Emergence of the direction selectivity map in adult animals occurs while the average response amplitude increases [juve- 
nile: $\operatorname{mean}_{k}(\langle(\Delta F / F)(k)\rangle)=0.02 \pm 0.04$, $N=2713$ neurons; adults: $\operatorname{mean}_{k}(\langle(\Delta F /$ $F)(k)\rangle)=0.04 \pm 0.08, N=3960$ neurons, where mean $_{k}$ is the mean over the directions $k=1-8$ ] but was not related to some important modifications of the directional tuning properties of the cell population. Figure $3 a$ shows in particular that the distribution of neuron tuning ratios were very similar for juvenile and adult animals. Mean \pm STD tuning ratios were indeed respectively equal to $0.50 \pm 0.20$ and $0.48 \pm 0.20$. The fraction of cells showing a significant tuning during the stimulation protocol was also similar between the two populations $(79 \%$ for the juvenile, $83 \%$ for the adult). As many stimuli (1200-1600) were presented during each protocol, adaptation could have occurred to a different extent in both ages. Therefore we also checked whether the observation of maps in adults only could be related to a difference of adaptation between juvenile and adult rats during the stimulation protocol. Adaptation during the stimulation protocol is represented for one neuron on Figure $3 b$, left, as the maximal response measured for each block of eight stimuli as a function of the block number $\left[\max _{k}(\langle(\Delta F / F)(k)\rangle)\right]$. The adaptation amplitude was defined as the relative variation of this maximal response between the first and the last block. The histogram of adaptation amplitude shown on Figure $3 b$, right, was similar between juvenile and adult animals [ $t$ test, $p>0.15$, mean variation in percentage \pm STD for juvenile $-6.8 \pm 24.3$ $(N=1792)$, for adult $-7.4 \pm 21.0(N=$ 2436)]. Therefore, the direction selectivity map emerges in adults and is related to the local reorganization of direction selectivities. It is due to neither a change in the population mean selectivities nor one in their adaptation properties.

\section{Pinwheel organization in adult but not in juvenile rats}

Visual inspection of the adult average map suggested that correlated microdomains are organized in a pinwheel-like pattern. To analyze the functional organization of the juvenile and adult maps, each neuron was described by its polar angular coordinate $\alpha$ and its preferred direction $\theta$ (Fig. $4 d$ ). Correlated $(\theta=\alpha)$, anti-correlated $(\theta=\alpha$ $+180^{\circ}$ ), or random models should be distinguishable in a raster plot of $\theta$ versus $\alpha$ and in the histograms of $|\theta-\alpha|$ (Andermann and Moore, 2006). In juvenile animals, no departure from a random model was apparent either in the raster plot or in the histogram (Fig. 4a, left). The circular-circular correlation coefficient (Fisher and Lee, 1983), measuring the extent to which data could be estimated from a linear association of the type $\theta= \pm \alpha+\alpha_{0}$, was not significantly different from zero $\left(\rho_{\mathrm{T}}=0.002, p=0.24\right)$. Surface blood vessels often cause nonuniform sampling of the

a
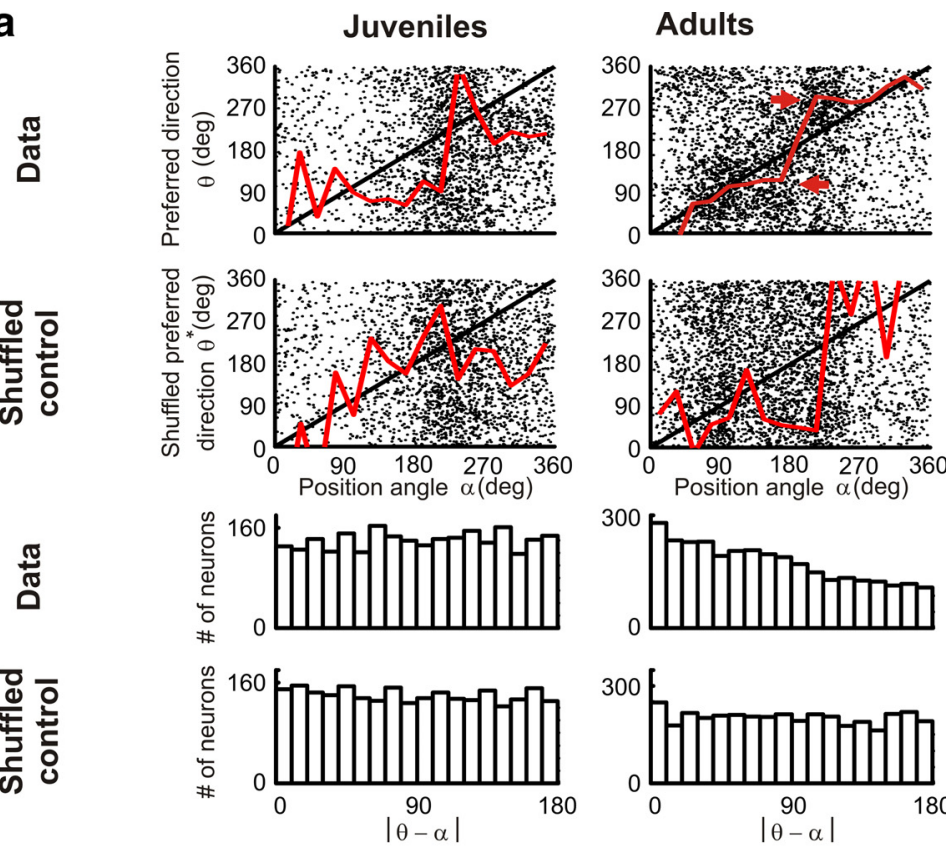
b

Pinwheel
center
optimization
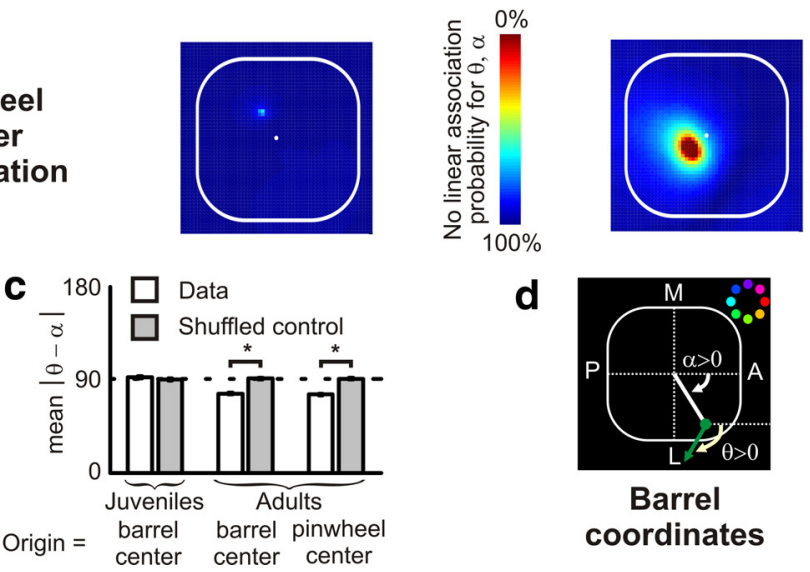

Figure 4. Pinwheel organization of average direction selectivity map phase-locked with somatotopy in adult rats. $\boldsymbol{a}$, Preferred direction $\theta$ versus polar angle coordinate $\alpha$ raster plots and probability distributions for $|\theta-\alpha|$ (shown in histogram form) for all significantly anisotropic neurons recorded in juveniles (left) or adults (right) and their associated shuffled control. Red solid line, Circular mean of $\theta$, averaged over $\pi / 8$ intervals of $\alpha$. Average values $\pm \pi$ around the principal diagonal (black solid line) are shown. Directions around the angles $110^{\circ}$ and $290^{\circ}$ were slightly overrepresented in adults, forming two short plateaus (red arrows). $\boldsymbol{b}$, Color-coded maps of the probability of no linear association between $\theta$ and $\alpha$ in juvenile (left) and adult (right) rats as a function of the origin of the polar coordinates. Probability values are shown at each point in the map for an $\alpha$ origin taken at this location (color code, central scale). c, Mean $|\theta-\alpha|$ values obtained for juvenile and adult rats with position angle referenced on barrel center, and in the case of adult rats also referenced on the spatial position found in $\boldsymbol{b}$ for maximal linear correlation between $\theta$ and $\alpha$. Light gray bars, Same analysis applied to shuffled controls. Only adult data were significantly (asterisks) below $90^{\circ}(p<$ $0.001)$. $\boldsymbol{d}$, Scheme showing a neuron (green dot) in a barrel column (borders, white lines) in normalized coordinates. Neuron polar coordinate, Position angle $\alpha$. Green arrow, Neuron preferred direction $\theta$, labeled according to color code. $\alpha=0^{\circ}$ and $\theta=0^{\circ}$ along the rostral direction, and for both, positive values are counted in clockwise rotation.

barrel section in our recordings, which could in turn create artificial correlation between $\alpha$ and $\theta$. Therefore we checked for data bias by shuffling randomly the preferred directions between neurons but keeping their angular position. If no bias appeared in our data, shuffled data should present a flat $|\theta-\alpha|$ histogram. This was indeed the case for the shuffled control of juvenile rats (Fig. $4 a$, left). Neurons in the raster plot of adults were more densely grouped around the diagonal $\theta=\alpha$ (Fig. 4a, right). Circularcircular correlation coefficient departed significantly from zero $\left(\rho_{\mathrm{T}}=0.029, p<0.00005\right)$, confirming that in this case $\theta$ and $\alpha$ were not independent. The histogram of $|\theta-\alpha|$ showed a marked 

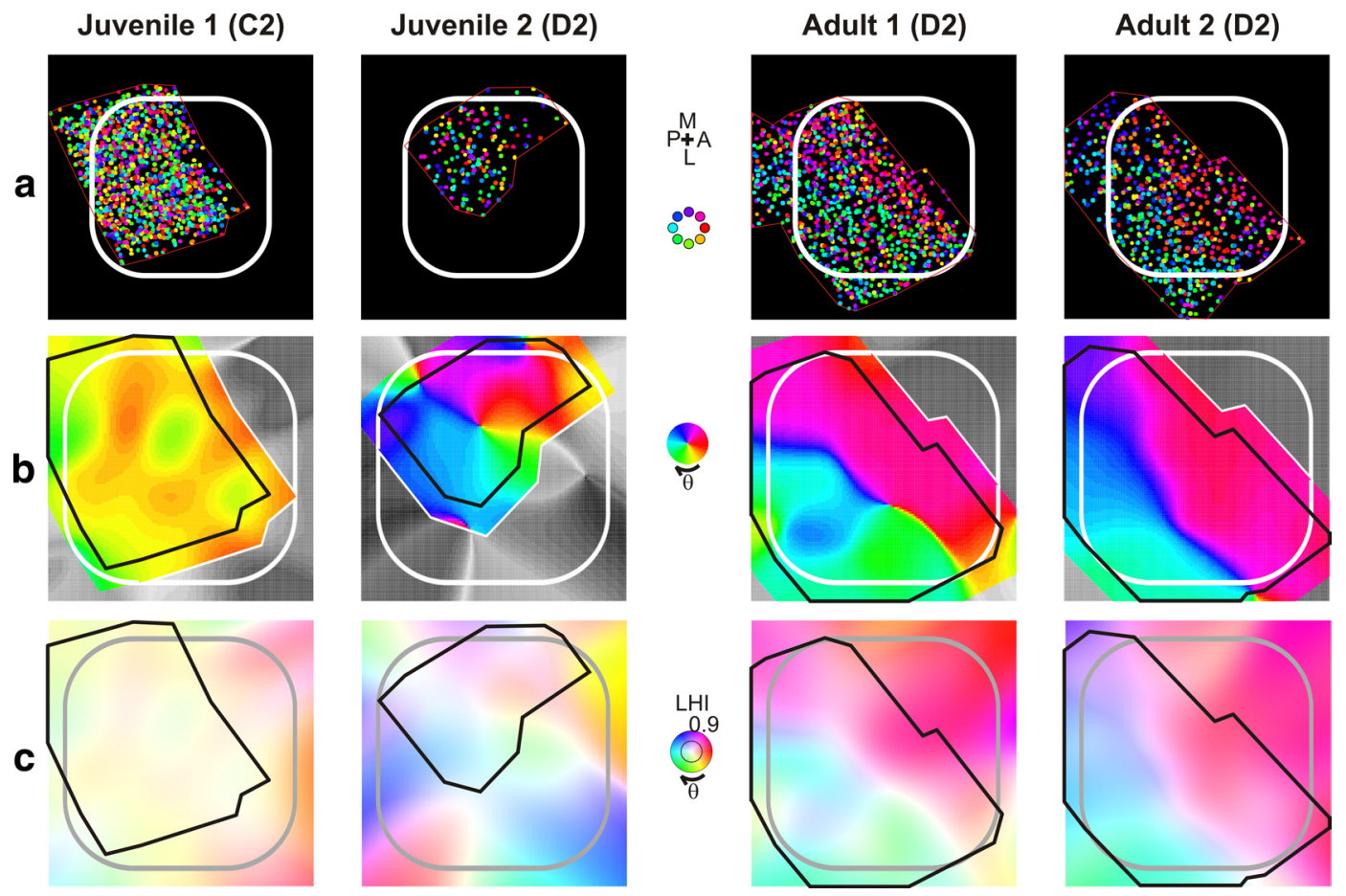

Figure 5. Direction selectivity maps for individual animals and barrels. Two juveniles (left panels) and two adults (right panels) are shown. $\boldsymbol{a}$, Raw maps for each rat. Red contour lines delimit the borders of the recorded zone. $\boldsymbol{b}$, Maps smoothed with a Gaussian filter (half-width $\omega=0.2$ in normalized barrel coordinates). Black lines, Contours of recorded zones. Poorly sampled areas distant of the recorded zone by a distance larger than $\omega$ are shown in grayscale. c, Same map with color saturation scaled according to local homogeneity index.

maximum around zero, indicating that the correlated model $\theta=$ $\alpha$ better described the adult data than anti-correlated or random models. A shuffled control for adults showed a flat histogram meaning that the observed correlation was not due to bias in our data (Fig. $4 a$, right). These analyses demonstrated that there was a dominant relation $\theta=\alpha$ common to all recorded barrels in the adult rats. This pinwheel structure was, however, distorted, with all preferred directions $\theta$ not represented with identical spatial angular intervals. An expanded representation of the directions around the opposing angles $110^{\circ}$ and $290^{\circ}$ along a tilted axis was evidenced by the two plateaus of the piecewise $\theta$ average (Fig. $4 a$, right).

\section{Pinwheel center located close to barrel center}

We explored whether the pinwheel center corresponded to the anatomic barrel central axis. By recalculating the probability for no linear association between $\theta$ and $\alpha$, but varying the origin of the polar coordinates used to determine $\alpha$, we searched for the pinwheel center that optimally fitted the correlated model to our data. A strong optimum was found only with adult data, slightly shifted toward the posterolateral side of the anatomical barrel center (Fig. 4b). The mean value for $|\theta-\alpha|$ is slightly reduced in adults when the optimum pinwheel center is taken as origin for polar coordinate $\alpha$ (pinwheel origin: mean value $=74.7^{\circ}$; barrel center origin: mean value $\left.=75.6^{\circ}\right)$. In both cases, the mean $\mid \theta-$ $\alpha \mid$ differed significantly from the mean value for the shuffled control (both $p<0.001$ ), which was not the case for juvenile rats $(p=0.98)$ (Fig. $4 c)$.

\section{Somatotopic principle of the pinwheel organization}

The spatial organization of SIbf provides a precise correspondence between coordinates in the external environment and on the cortical surface. How the pinwheel directional map present in adults is linked to these somatotopic coordinates can be addressed by our data. Indeed, $\theta$ and $\alpha$ measured in adult rats in Figure $4 a$ were not only significantly linearly associated, but their relationship $\theta=\alpha+\alpha_{0}$ had an offset $\alpha_{0}=-1.1 \pm 6.0^{\circ}(95 \%$ confidence interval). $\theta$ and $\alpha$ were thus in phase with no significant shift: neurons preferring a deflection toward an adjacent vibrissa tend to be preferentially localized closest to this adjacent vibrissa barrel. The vibrissa direction selectivity map is superposed on the "barrel" map, obeying a precise correspondence between each other, which is shaped by the coordinates in the external environment.

Direction selectivity maps are also present in a single barrel of individual animals

In several experiments, a sufficiently large portion of the barrel column was accessible such that a direction selectivity map could be recorded for a single barrel in an individual animal. A disordered juxtaposition of neurons with apparently random preferred directions was visible in juveniles (Fig. $5 a$ ). It indicates that the lack of spatial organization observed in the averaged map (Fig. 2c, left) is not the result of mixing unrelated animals or barrels together but is rather a property of individual barrels and animals. The spatial segregation in a pinwheel-like collection of domains was present not only in the adult averaged map (Fig. $2 c$, right) but also in individual maps (Fig. 5a). After smoothing, pinwheel-like structures became apparent (Fig. $5 b$ ). These pinwheels did not exhibit a perfect radial symmetry, and domains corresponding to anteroposterior directions were overrepresented. Sharp frontiers between rostral and caudal preference domains, $\sim 100 \mu \mathrm{m}$ wide, were visible (Fig. $5 b$ ), although they appeared ill defined when examined in the original raw maps (Fig. 5a). Finally, the LHI averaged over each recorded neuronal position remained significantly smaller in the juveniles (juvenile 
1: $\overline{\mathrm{LHI}}_{J, 1}=0.140 \pm 0.001$; juvenile 2 : $\left.\overline{\mathrm{LHI}}_{J, 2}=0.139 \pm 0.006\right)$ than in the adults (adult 1: $\overline{\mathrm{LHI}}_{A, 1}=0.242 \pm 0.003$; adult 2: $\overline{\mathrm{LHI}}_{A, 2}=0.335 \pm 0.004$; all differences between $\overline{\mathrm{LHI}}_{J}$ and $\overline{\mathrm{LHI}}_{A}$ significant with $p<0.0001)$. This confirmed that any structure present in individual juvenile maps remained much more disordered than the structure observed in adults (Fig. $5 c$ ).

\section{Neurons and local neuropil have correlated preferred directions} Calcium activity at neuronal somata reflects the spiking activity of cells (Kerr et al., 2005), i.e., the outputs of the local neuronal circuits. Their inputs can be estimated from the neuropil calcium signal, which is determined by axonal structures around neurons (Kerr et al., 2005). We next address the question of whether the neuropil also exhibits a segregated spatial structure and whether its evolution with age parallels that found for the neuronal outputs. Typical neuropil fields in juveniles and adults are presented in Figure 6, $a$ and $b$. The agreement between local input and output directional selectivities was evaluated by the correlation between the preferred directions of every neuron and its surrounding neuropil. Cumulative histograms showed that any neuron-neuropil correlation in the range -1 and +1 could be found in juvenile and adult fields with an excess of strongly correlated couples (juvenile: $27 \pm 11 \%$ of neurons having $>0.9$ correlation with their nearest neuropil patch, $N=2040$ neurons, 29 fields, mean $\pm \mathrm{SD}$ across fields; adult: $36 \pm 12 \%$ of neurons $>0.9$ correlation, $N=4908$ neurons, 41 fields; significantly more frequently in adults than in juveniles; $p=0.019$ ) (Fig. $6 c, d$ ). This is unlikely to arise from a contamination of the neuron fluorescence by the neuropil (Göbel and Helmchen, 2007). To separate neuron and neuropil signals, great care was taken to optimize the in-plane and axial resolutions of the microscope and to define conservative ROI at neuronal somata (Göbel and Helmchen, 2007; Kerr et al., 2007) (recordings were included in the analysis of a neuron only when they occurred at the depth of its equatorial plane $\pm 3 \mu \mathrm{m}$, Materials and Methods). Absence of contamination was assessed by inspecting the nonsystematic co-occurrence of calcium transients in neurons and neuropil (Fig. 1d). Moreover, approximately one-third of the neurons and corresponding neuropil had no correlation between their preferred directions or were even anti-correlated, demonstrating that the fluorescence signals from both structures have been separated properly (juvenile: $32 \pm 13 \%$ of neurons-neuropil having negative correlation, 29 fields; adult: $26 \pm 13 \%$, 41 fields, mean \pm SD across fields). homogeneous directions.
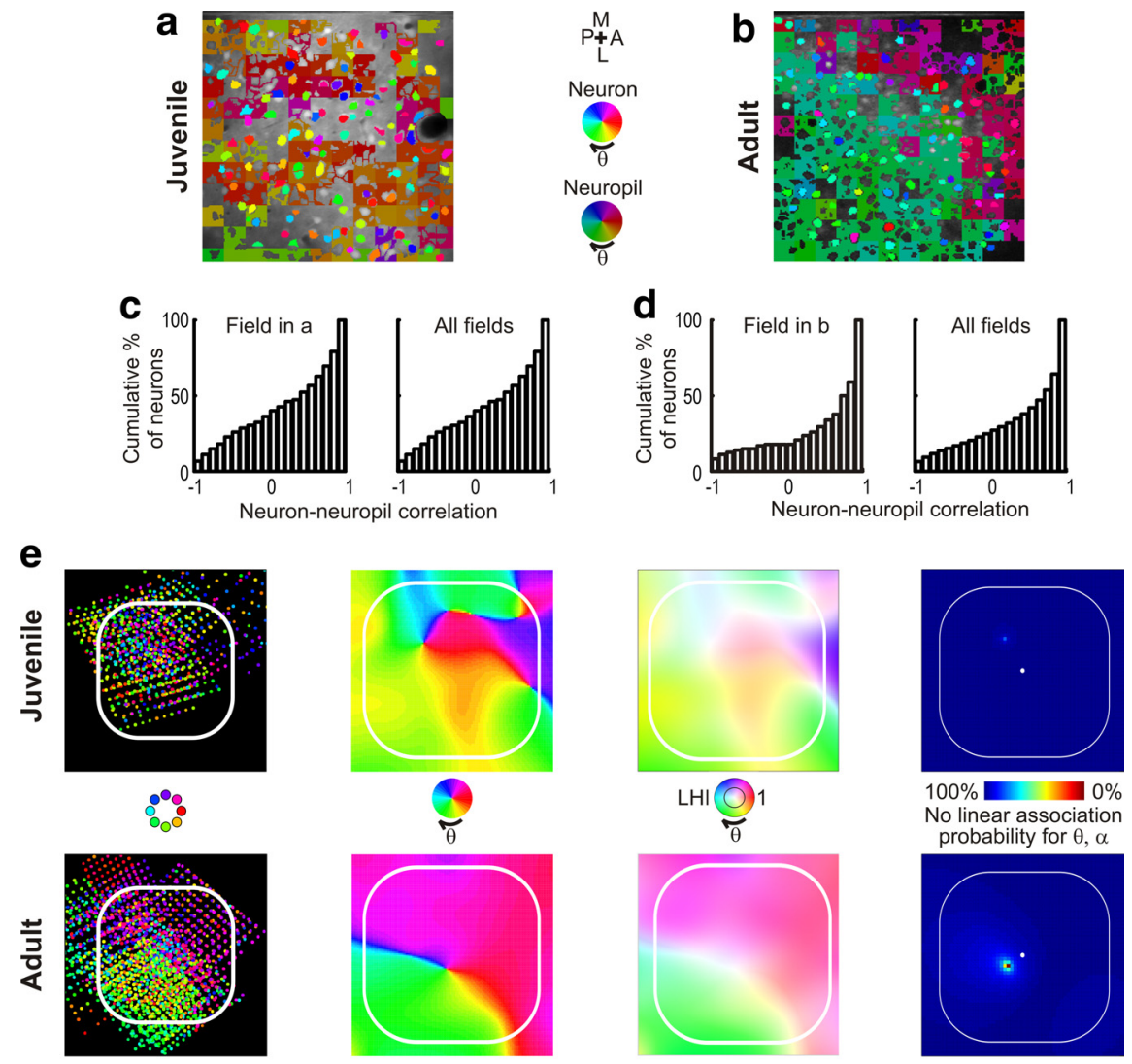

$100 \%$ probability for $\theta$,
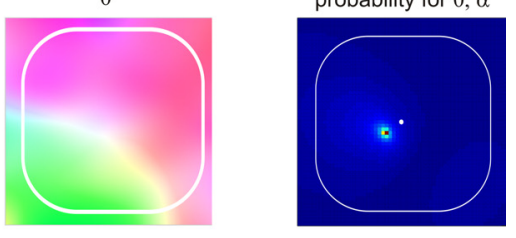

f

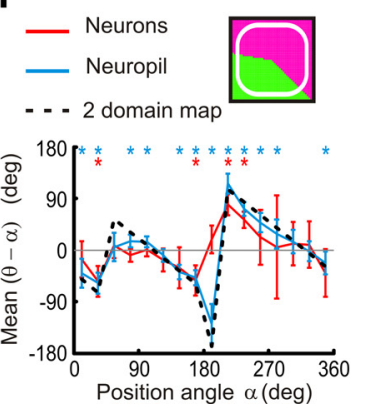

g

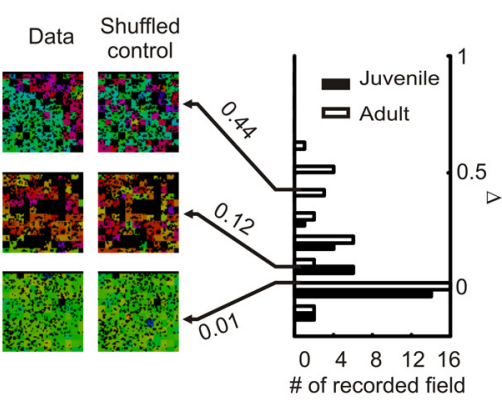

Figure 6. Spatial organization of neuropil direction selectivity. $\boldsymbol{a}, \boldsymbol{b}$, Examples of fields in juvenile $(\boldsymbol{a})$ and adult $(\boldsymbol{b})$ rats with color coded indication of preferred directions of significantly anisotropic neurons and neuropil patches ( $25 \mu \mathrm{m} \times 25 \mu \mathrm{m})$ (same fields as in Fig. 2a). c, d, Cumulative histograms of the correlation between a neuron and its surrounding neuropil. c, Juvenile; $\boldsymbol{d}$, adult. In each case, data are shown for the fields in $\boldsymbol{a}$ and $\boldsymbol{b}$ (left) and averaged over all fields (right). $\boldsymbol{e}$, Neuropil direction selectivity maps obtained by superposition of all fields in juvenile (upper raw) and in adult (lower row) rats. From left to right, Significantly anisotropic neuropil patches are represented by colored dots; smoothed maps with a Gaussian filter $(\omega=0.2)$; same smoothed maps but with color saturation scaled by the local homogeneity index; and color-coded maps of the probability of no linear association between $\theta$ and $\alpha$ as a function of the origin of the polar coordinates. $\boldsymbol{f}$, Mean difference between the direction selectivity $\theta$ of neurons (red) or neuropil (blue) and the position angle $\alpha$ (origin: barrel center) in all adult rats. Neurons and neuropil patches are pooled by $22.5^{\circ}$-wide bins of $\alpha$. A perfect pinwheel would correspond to the zero baseline (gray line). Neuropil deviates significantly over a larger fraction of the barrel section from a perfect pinwheel than neurons (asterisks above each bin if $p<0.05)$. The mean $(\theta-\alpha)$ for neuropil resembles that of a pattern of two uniform domains with preferred directions of $110^{\circ}$ and $320^{\circ}$ (inset) [black dotted line, corresponding to mean $(\theta-\alpha)$ ]. $\boldsymbol{g}$, Histogram of the metric $\Delta$ calculated on every field. Three examples of fields (data, left) and associated shuffled controls (right) are shown, with their $\Delta$ value (arrows) and their position in the histogram. Two upper fields, Same fields as in $\boldsymbol{a}$ and $\boldsymbol{b}$. Lower field, Example of an adult field entirely filled with near-

Neuropil direction selectivity map forms a pinwheel-like structure in adults

By combining all results obtained across animals, averaged neuropil direction selectivity maps could be formed (Fig. 6e). No particular spatial organization could be extracted in juvenile rats, whereas a pinwheel-like structure was obvious for adults. As for 
a

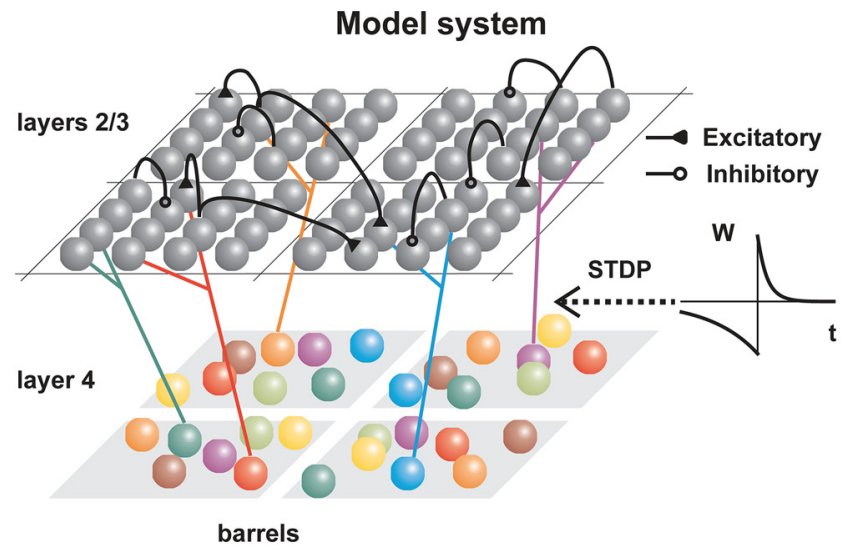

b

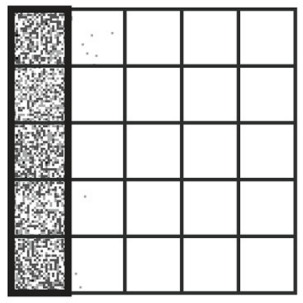

Sensory experience

C

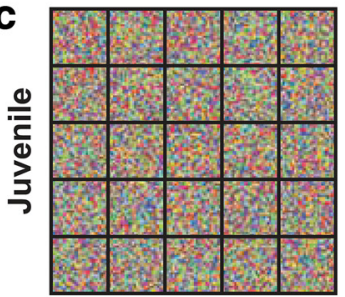

d

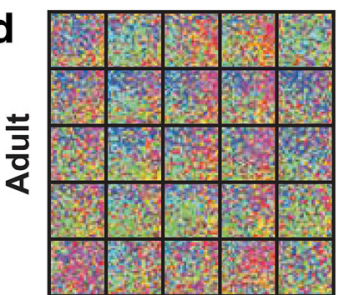

e
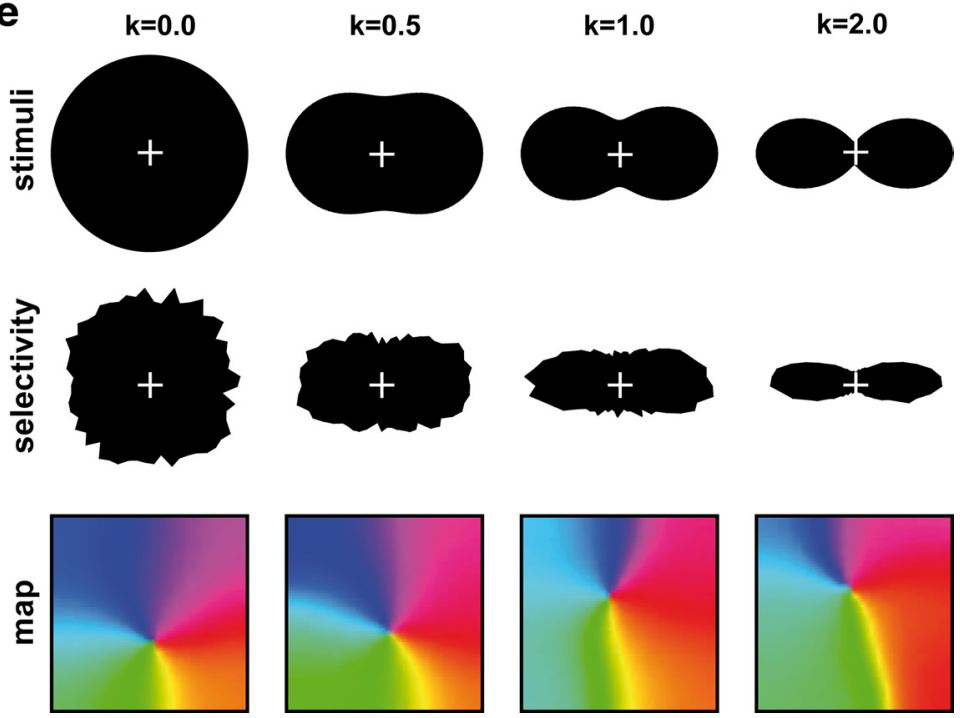

Figure 7. Emergence of a pinwheel organization in a numerical model of the barrel cortex network. $\boldsymbol{a}$, Scheme showing the two-layer model network. The synaptic weights between layer 4 and layer $2 / 3$ excitatory neurons are governed by an STDP rule. $\boldsymbol{b}$, the neuron map, we found only with adult data an optimum for the probability for no linear association between $\theta$ and $\alpha$, as a function of the polar coordinates used to define $\alpha$. The center of the adult neuropil map determined by this method was slightly shifted toward the posterolateral side of the anatomical barrel center (Fig. $6 e$ ), as for the adult neuron map. When compared to the neuron map in Figure 2, $e$ and $f$, the pinwheel structure of the neuropil map in adults tended to be less regular: the directions around the opposing angles $110^{\circ}$ or $320^{\circ}$ encompass larger fractions of the pinwheel than the directions around $45^{\circ}$ or $225^{\circ}$. We tested for significant differences between $\theta$ and $\alpha$, as a function of $\alpha$ in $22.5^{\circ}$ increments (Fig. $6 f)$. This revealed a deviation from the regular pinwheel, which was more pronounced for the neuropil (12/16 angle bins displayed a significant difference) than for neurons $(4 / 16)$. The curve mean $(\theta-\alpha)$ for adult neuropil was similar to that expected for a two-domain map (Fig. $6 f$, inset). The neuropil direction selectivity map thus resembled a distorted pinwheel dominated by two half planes representing almost opposite directions along a tilted dorsocaudal-ventrorostral axis.

A two-domain map exhibits a characteristic discontinuity along a linear frontier. We next examined the sharpness of this frontier. As the average adult neuropil map could be limited in spatial resolution by the process of referencing two-photon and histological images, we analyzed directly whether sharp frontiers could also

\section{$\leftarrow$}

Spikes produced by layer $2 / 3$ neurons when a bar is swept across the vibrissa pad (each frame is separated by $5 \mathrm{~ms}$ ). Activation of the first barrels (left) induces suppression in neighboring neurons, on the left side of barrels that are subsequently activated (middle and right). This results in movement-induced correlations between direction of vibrissa deflections and position of activated neurons within barrels. $C$, Initial condition (juvenile rats) of the direction selectivity map in layer $2 / 3$ for $5 \times 5$ barrels arranged as a square grid. Direction preferences were attributed randomly to neurons. Left, Each colored dot represents one neuron with its color coded preferred direction. Middle and right, Maps smoothed with a Gaussian filter without and with color saturation scaled according to local homogeneity index. $\boldsymbol{d}$, Same direction selectivity maps after learning (adult rats), as in c. $\boldsymbol{e}$, Relationship between final selectivity and stimuli presented during learning. Top, Distributions of presented bar directions in polar representation. The first distribution is unbiased $(k=0)$, while other distributions are increasingly biased toward rostrocaudal directions $(k>0)$. Middle, Distributions of preferred directions of $L 2 / 3$ neurons after learning. Bottom, Direction selectivity map of the central barrel after learning, as in $\boldsymbol{c}$ and $\boldsymbol{d}$. 
be found in adult single fields. We computed a metric $\Delta$ inspired from the geometrical problem of the minimization of the interface cost between two juxtaposed domains (Materials and Methods). $\Delta$ values close to 1 reveal the presence of a sharp frontier, whereas $\Delta$ values close to 0 indicate an absence of such a frontier. The histogram of $\Delta$ was computed from all fields recorded in adult and juvenile rats (Fig. $6 g$ ). High values for $\Delta$ only occurred in adult animals, showing that sharp frontiers were a characteristic of adult rats. They were typically one neuropil patch wide (25 $\mu \mathrm{m})$, i.e., of a scale comparable to the interneuronal distance.

\section{Learning of stereotyped multivibrissa deflections may contribute to the formation of the direction selectivity barrel map}

Our data demonstrated that the superposition of the adult neuron or neuropil direction selectivity maps with the barrel map obeys a precise correspondence, shaped by the coordinates in the external environment. This suggests that mechanisms involving contact experience with objects could be determinant for the emergence of the maps. Since their formation occurs during the adulthood, requiring processes still in action at that age, we investigated with a numerical simulation whether synaptic plasticity mechanisms within SIbf could be at their origin. In our simulation, the link between direction selectivity and somatotopy is due to repetitive contacts with objects crossing the vibrissa pad in random directions, therefore learning to associate local deflection of a vibrissa with the precisely timed subsequent activation of adjacent vibrissae.

The barrel cortex neuronal network was modeled as a twolayer (L4 and L2/3) system with plastic synaptic connections obeying STDP rules between L4 and L2/3 excitatory neurons (Fig. $7 a$ ). In layer $2 / 3$, inhibitory connections were assumed to have a shorter length scale than excitatory ones. L4 neurons were assumed to be directionally selective (Bruno et al., 2003; Lee and Simons, 2004; Andermann and Moore, 2006). After implementing uniform random connectivity between $\mathrm{L} 4$ and $\mathrm{L} 2 / 3$, the simulated integrate-and-fire L $2 / 3$ neurons thus received a collection of plastic and directionally selective feedforward inputs.

The starting point of the simulation was a layer $2 / 3$ composed of direction selective neurons without any spatial organization of the selectivities (Fig. $7 c$ ), as observed in juvenile animals. We explored the evolution of that network in presence of stimulations consisting of bars sliding across the vibrissa pad, which deflected vibrissae in a regular spatio-temporal pattern (Fig. 7b). Training using this protocol induced modifications of synaptic weights at L4 to L2/3 synapses and of neuronal selectivities, according to the following principal steps: (1) when a vibrissa was deflected, the corresponding barrel column was activated and neighboring columns were suppressed due to lateral inhibition; (2) when the next vibrissa was deflected, the corresponding barrel column was activated but neurons near the previous barrel column remained less active because of inhibition; (3) with STDP, those neurons that were not suppressed experienced a potentiation of their synapses that was selective for the presented direction. Therefore, when the stimulating bar moved across the vibrissa pad, a wave of inhibition propagated from barrel to barrel, preferentially inactivating in each barrel column the side first reached by the wave. This pattern of activity within the column was associated with the direction of deflection by the STDP plasticity. At the end of the training, neurons were spatially segregated on average according to their direction preferences, as observed in the raw and in the filtered data (Fig. 7d). Quantitatively, the distribution of preferred directions is determined by the statistics of stimuli during the learning period (Fig. 7e). When we introduced a bias for rostrocaudal directions in presented bars, we found the same bias in the final distribution of preferred directions. This suggests that the preference for rostrocaudal directions seen experimentally in adult animals could result from an exposure to natural stimulations with a rostrocaudal bias. This model shows that repetitive presentation of stimuli across the vibrissa pad with temporal correlations could account for the reorganization of the selectivity map within a barrel column toward a clustering of neurons sharing similar direction selectivities.

\section{Discussion}

We have demonstrated the surprisingly late emergence of the spatial organization of neuronal directional selectivity in the upper layers of the rat barrel cortex long after all known critical periods in the somatosensory system. The development of most known sensory cortical maps occurs very early, usually before the first postnatal month in rodents. In layer 4 , the barrel field organization is established a few days after birth (Inan and Crair, 2007; Petersen, 2007), and the map of layer $2 / 3$ receptive fields for vibrissa stimulation is formed at 2 weeks postnatal (Stern et al., 2001). The same precocity of map development is observed for other sensory modalities. In the mouse visual cortex, maps for visual space and for ocular dominance are already observed at eye opening (9 d) (Smith and Trachtenberg, 2007). Similarly, an adult-like tonotopic map of sound frequency in the rat auditory cortex AI is established at 3 weeks postnatal (Zhang et al., 2002). More refined maps, absent in rodents but present in other animals, also appear early. In the cat visual cortex, the orientation map is formed before the end of the second postnatal week (Crair et al., 1998), and in the ferret visual cortex, the direction selectivity map is observed just after the time of eye opening (Li et al., 2008). Compared to all these cortical maps, the barrel cortex direction selectivity map emerges remarkably late.

It is therefore natural to question whether plasticity on its own, which is maintained throughout life in the cortex (Shulz and Frégnac, 1992; Karmarkar and Dan, 2006; Petersen, 2007), could be the basis of the formation of the direction selectivity tactile map. Activity-dependent plasticity has indeed been demonstrated in SIbf both at the cellular level (Glazewski and Fox, 1996; Jacob et al., 2008) and at the scale of the entire barrel (Feldman and Brecht, 2005; Frostig, 2006). Sensory activity is known to shape and strengthen most sensory cortical maps such as those for ocular dominance in the rodent visual cortex (Berardi et al., 2000; Smith and Trachtenberg, 2007) or for orientation and direction selectivity in the cat and ferret visual cortex (Crair et al., 1998; Li et al., 2008). The aim of our numerical simulations was to investigate how experience in tactile contacts could modify the spatial organization of neuronal direction selectivity in SIbf via synaptic plasticity.

In our simulations, we start with neurons in layer $2 / 3$ already tuned in direction but randomly distributed in the barrel column to examine the transition observed experimentally between juvenile and adult animals. The origin of the random distribution could come from the late maturation of whisking, requiring $\sim 1$ month (Welker, 1964; Landers and Philip Zeigler, 2006), which could reduce drastically the correlated stimulations received by juvenile animals. We verified by simulation that training our model with uncorrelated multivibrissa sequences and starting with an initial population of directionally nonselective layer $2 / 3$ neurons produces tuned neurons distributed randomly, similarly to those observed in juveniles. Our model predicted with success 
the emergence of the pinwheel structure and showed that synaptic plasticity on its own can be at the origin of the spatial reorganization of directional selectivity in mature animals.

Our model describes the contact with an object by the sequence of successive vibrissa activation. The introduction of time as a parameter in our description enables us to relate the plastic reorganization to the mechanism of STDP. Another Hebbian learning model was recently proposed to account for the development of a direction map in the barrel cortex (Wilson et al., 2010). Our model differs in two ways. First, while the model of Wilson et al. (2010) was a relatively abstract rate-based model, our model uses spiking neuron models with realistic physiological data (time constants, EPSP and IPSP amplitude, synaptic connectivity, etc.). Therefore, in addition to providing a mechanistic explanation of the development of direction maps, it also shows that this explanation is compatible with the known physiology of the barrel cortex. Second, the mechanism of map formation is slightly different. In the model of Wilson et al. (2010), map formation relies only on spatial correlations, with movement playing no role: a direction map emerges because of the assumed relationship between the shape of an object and the angle by which vibrissae are deflected. Instead, our model relies on spatiotemporal correlations: a direction map emerges because movement direction correlates with deflection direction. We believe this is more robust because vibrissa deflection should be better correlated with movement than with object shape, when friction is taken into account.

Our findings reconcile the apparently contradictory results from two previous mapping studies (Andermann and Moore, 2006; Kerr et al., 2007), as they reveal that the main cause of their discrepancy was the age of the animals. The pinwheel in adult rats had received additional support with recent intrinsic optical imaging measurements in 2- to 4-month-old rats (Tsytsarev et al., 2010). However, only two-photon microscopy could provide insight into the fine detail of the pinwheel structure and revealed a mosaic of neurons with marked variations in selectivity between neighbors, approaching only on average the map obtained from smoothed electrophysiological data (Andermann and Moore, 2006).

In the smoothed neuronal map, some directions occupy larger fractions of the barrel. This irregular distribution was more pronounced for the neuropil map. The preferred direction of a neuropil patch reflects the selectivity of the majority of axons terminals in the patch (Kerr et al., 2005). As it mixes selective terminals from layer 4 and spatially segregated terminals of layer $2 / 3$ horizontal connections, the neuropil activity follows the neuronal direction selectivity map and accentuates with a "winner takes all" effect the irregular angle distribution with dominant dorsocaudal and ventrorostral directions. It corroborates the rostrocaudal bias previously reported in adults (Andermann and Moore, 2006) and could be interpreted as resulting from an increased experience in the directions explored during whisking. However, let us note that the observed irregular distributions of preferred angles could also partially result from slicing the cortex with a small angle with respect to the cortical layers.

What would be the benefit for the existence of the direction selectivity map in the rat barrel cortex? By analogy with the visual system, the rat could use the unique pattern of activity in SIbf elicited by the deflection of two adjacent vibrissae in opposite directions to encode the contour of a palpated object. Thus the barrel cortex maps refinement through sensory learning might improve the discrimination performance of the rat in complex multivibrissa tactile tasks.

\section{References}

Andermann ML, Moore CI (2006) A somatotopic map of vibrissa motion direction within a barrel column. Nat Neurosci 9:543-551.

Andermann ML, Ritt J, Neimark MA, Moore CI (2004) Neural correlates of vibrissa resonance; band-pass and somatotopic representation of highfrequency stimuli. Neuron 42:451-463.

Bartfeld E, Grinvald A (1992) Relationships between orientation-preference pinwheels, cytochrome oxidase blobs, and ocular-dominance columns in primate striate cortex. Proc Natl Acad Sci U S A 89:11905-11909.

Benninger RK, Ashby WJ, Ring EA, Piston DW (2008) Single-photoncounting detector for increased sensitivity in two-photon laser scanning microscopy. Opt Lett 33:2895-2897.

Berardi N, Pizzorusso T, Maffei L (2000) Critical periods during sensory development. Curr Opin Neurobiol 10:138-145.

Brecht M (2007) Barrel cortex and whisker-mediated behaviors. Curr Opin Neurobiol 17:408-416.

Bruno RM, Khatri V, Land PW, Simons DJ (2003) Thalamocortical angular tuning domains within individual barrels of rat somatosensory cortex. J Neurosci 23:9565-9574.

Crair MC, Gillespie DC, Stryker MP (1998) The role of visual experience in the development of columns in cat visual cortex. Science 279:566-570.

Dunn AK, Wallace VP, Coleno M, Berns MW, Tromberg BJ (2000) Influence of optical properties on two-photon fluorescence imaging in turbid samples. Appl Opt 39:1194-1201.

Feldman DE, Brecht M (2005) Map plasticity in somatosensory cortex. Science 310:810-815.

Feldmeyer D, Lübke J, Silver RA, Sakmann B (2002) Synaptic connections between layer 4 spiny neurone-layer $2 / 3$ pyramidal cell pairs in juvenile rat barrel cortex: physiology and anatomy of interlaminar signalling within a cortical column. J Physiol 538:803-822.

Feldmeyer D, Lübke J, Sakmann B (2006) Efficacy and connectivity of intracolumnar pairs of layer $2 / 3$ pyramidal cells in the barrel cortex of juvenile rats. J Physiol 575:583-602.

Fisher NI, Lee AJ (1983) A correlation coefficient for circular data. Biometrika 70:327-332.

Fisher NI, Lee AJ (1986) Correlation coefficients for random variables on a unit sphere or hypersphere. Biometrika 73:159-164.

Frostig RD (2006) Functional organization and plasticity in the adult rat barrel cortex: moving out-of-the-box. Curr Opin Neurobiol 16:445-450.

Gibson JM, Welker WI (1983) Quantitative studies of stimulus coding in first-order vibrissa afferents of rats. 1. Receptive field properties and threshold distributions. Somatosens Res 1:51-67.

Glazewski S, Fox K (1996) Time course of experience-dependent synaptic potentiation and depression in barrel cortex of adolescent rats. J Neurophysiol 75:1714-1729.

Göbel W, Helmchen F (2007) In vivo calcium imaging of neural network function. Physiology (Bethesda) 22:358-365.

Goodman DF, Brette R (2009) The brian simulator. Front Neurosci 3:192-197.

Helmchen F, Denk W (2005) Deep tissue two-photon microscopy. Nat Methods 2:932-940.

Helmstaedter M, Sakmann B, Feldmeyer D (2009) Neuronal correlates of local, lateral, and translaminar inhibition with reference to cortical columns. Cereb Cortex 19:926-937.

Inan M, Crair MC (2007) Development of cortical maps: perspectives from the barrel cortex. Neuroscientist 13:49-61.

Jacob V, Brasier DJ, Erchova I, Feldman D, Shulz DE (2007) Spike timingdependent synaptic depression in the in vivo barrel cortex of the rat. J Neurosci 27:1271-1284.

Jacob V, Le Cam J, Ego-Stengel V, Shulz DE (2008) Emergent properties of tactile scenes selectively activate barrel cortex neurons. Neuron 60:1112-1125.

Kapfer C, Glickfeld LL, Atallah BV, Scanziani M (2007) Supralinear increase of recurrent inhibition during sparse activity in the somatosensory cortex. Nat Neurosci 10:743-753.

Karmarkar UR, Dan Y (2006) Experience-dependent plasticity in adult visual cortex. Neuron 52:577-585.

Kerr JN, Greenberg D, Helmchen F (2005) Imaging input and output of neocortical networks in vivo. Proc Natl Acad Sci U S A 102:14063-14068.

Kerr JN, de Kock CP, Greenberg DS, Bruno RM, Sakmann B, Helmchen F (2007) Spatial organization of neuronal population responses in layer 2/3 of rat barrel cortex. J Neurosci 27:13316-13328. 
Landers M, Philip Zeigler H (2006) Development of rodent whisking: trigeminal input and central pattern generation. Somatosens Mot Res 23:1-10.

Lee SH, Simons DJ (2004) Angular tuning and velocity sensitivity in different neuron classes within layer 4 of rat barrel cortex. J Neurophysiol 91:223-229.

Lefort S, Tomm C, Floyd Sarria JC, Petersen CC (2009) The excitatory neuronal network of the $\mathrm{C} 2$ barrel column in mouse primary somatosensory cortex. Neuron 61:301-316.

Li Y, Van Hooser SD, Mazurek M, White LE, Fitzpatrick D (2008) Experience with moving visual stimuli drives the early development of cortical direction selectivity. Nature 456:952-956.

Lübke J, Feldmeyer D (2007) Excitatory signal flow and connectivity in a cortical column: focus on barrel cortex. Brain Struct Funct 212:3-17.

Nimmerjahn A, Kirchhoff F, Kerr JN, Helmchen F (2004) Sulforhodamine 101 as a specific marker of astroglia in the neocortex in vivo. Nat Methods $1: 31-37$.

Petersen CC (2007) The functional organization of the barrel cortex. Neuron 56:339-355.

Petersen CC, Sakmann B (2001) Functionally independent columns of rat somatosensory barrel cortex revealed with voltage-sensitive dye imaging. J Neurosci 21:8435-8446.

Sarid L, Bruno R, Sakmann B, Segev I, Feldmeyer D (2007) Modeling a layer 4-to-layer 2/3 module of a single column in rat neocortex: interweaving in vitro and in vivo experimental observations. Proc Natl Acad Sci U S A 104:16353-16358.

Shulz D, Frégnac Y (1992) Cellular analogs of visual cortical epigenesis. II. Plasticity of binocular integration. J Neurosci 12:1301-1318.
Smith SL, Trachtenberg JT (2007) Experience-dependent binocular competition in the visual cortex begins at eye opening. Nat Neurosci 10:370-375.

Song S, Miller KD, Abbott LF (2000) Competitive Hebbian learning through spike-timing-dependent synaptic plasticity. Nat Neurosci 3:919-926.

Stern EA, Maravall M, Svoboda K (2001) Rapid development and plasticity of layer 2/3 maps in rat barrel cortex in vivo. Neuron 31:305-315.

Stosiek C, Garaschuk O, Holthoff K, Konnerth A (2003) In vivo two-photon calcium imaging of neuronal networks. Proc Natl Acad Sci U S A 100:7319-7324

Sun QQ, Huguenard JR, Prince DA (2006) Barrel cortex microcircuits: thalamocortical feedforward inhibition in spiny stellate cells is mediated by a small number of fast-spiking interneurons. J Neurosci 26:1219-1230.

Tsytsarev V, Pope D, Pumbo E, Garver W (2010) Intrinsic optical imaging of directional selectivity in rat barrel cortex: application of a multidirectional magnetic whisker stimulator. J Neurosci Methods 189:80-83.

Welker WI (1964) Analysis of sniffing of the albino rat. Behaviour 22:223-244.

Wier WG, Balke CW, Michael JA, Mauban JR (2000) A custom confocal and two-photon digital laser scanning microscope. Am J Physiol Heart Circ Physiol 278:H2150-H2156.

Wilson SP, Law JS, Mitchinson B, Prescott TJ, Bednar JA (2010) Modeling the emergence of whisker direction maps in rat barrel cortex. PLoS One 5:e8778.

Zhang LI, Bao S, Merzenich MM (2002) Disruption of primary auditory cortex by synchronous auditory inputs during a critical period. Proc Natl Acad Sci U S A 99:2309-2314. 